\title{
Influence of argon and oxygen on charge-state-resolved ion energy distributions of filtered aluminum arcs
}

\author{
Johanna Rosén \\ Materials Chemistry, RWTH Aachen University, D-52056 Aachen, Germany \\ André Anders \\ Lawrence Berkeley National Laboratory, Berkeley, California 94720, USA \\ Stanislav Mráz, Adil Atiser, and Jochen M. Schneider \\ Materials Chemistry, RWTH Aachen University, D-52056 Aachen, Germany
}

\begin{abstract}
The charge-state-resolved ion energy distributions (IEDs) in filtered aluminum vacuum arc plasmas were measured and analyzed at different oxygen and argon pressures in the range $0.5-8.0$ mTorr. A significant reduction of the ion energy was detected as the pressure was increased, most pronounced in an argon environment and for the higher charge states. The corresponding average charge state decreased from 1.87 to 1.0 with increasing pressure. The IEDs of all metal ions in oxygen were fitted with shifted Maxwellian distributions. The results show that it is possible to obtain a plasma composition with a narrow chargestate distribution as well as a narrow IED. These data may enable tailoring thin-
\end{abstract}


film properties through selecting growth conditions that are characterized by predefined charge state and energy distributions. 


\section{INTRODUCTION}

Vacuum arc plasma is well known to be highly ionized with comparatively high directed ion energies. The average ion energy is material dependent and ranges from 20 to $150 \mathrm{eV},{ }^{1}$ though the ion energy distributions (IEDs) extend up to several hundreds of $\mathrm{eV}$. $^{2}$ The ion charge states $\left(Q_{\mathrm{i}}\right)$ are important for plasma processing applications that utilize substrate bias, since the kinetic energy gain $\left(\Delta E_{i}\right)$ across the potential difference between the plasma and the substrate $(\Delta U)$ is given by $\Delta E_{i}=Q_{i} \cdot e \cdot \Delta U$. It is well known that the kinetic ion energy, and therefore also the charge states, affect the microstructure evolution, and hence the film properties. ${ }^{3-5}$ Precise control of the kinetic ion energy during film growth requires narrow charge state distributions (CSDs) as well as narrow IEDs. These requirements are in contrast to what is generally measured for vacuum arc plasmas. Aluminum plasma, for example, contains ions with charge states of up to three, and the IEDs extend up to $250 \mathrm{eV} .{ }^{6}$ It is known that through the introduction of background gas, these plasma properties can be influenced. Reduced ion charge states ${ }^{7-10}$ and ion energies ${ }^{7,11-12}$ have been reported when the pressure was increased. However, most studies deal with average charge states, ${ }^{7,10}$ average energies, ${ }^{7,12}$ or IEDs averaged over all ion species present. ${ }^{11}$ Detailed information about the effect of gas on individual charge states including their IEDs is limited: Chhowalla analyzed charge-state-resolved IEDs for zirconium arc plasma before and after introduction of nitrogen, ${ }^{13}$ and Bilek et al. reported on the influence of the nitrogen partial pressure on IEDs of $\mathrm{Ti}^{+}{ }^{+}{ }^{14}$ Furthermore, Strauss et al. investigated the effect of nitrogen pressure on the 
energy range of the principal ions in TiN deposition. ${ }^{15} \mathrm{~A}$ study including the effects of pressure on the charge state resolved IEDs of the full metal plasma composition has previously not been reported. However, it is straight forward to understand that knowing the "complete" plasma composition and all IEDs are prerequisites for being able to understand microstructure evolution.

In this article, the plasma chemistry and energetics of an aluminum arc operating at vacuum base pressure as well as in oxygen and argon atmospheres are presented and discussed. The charge state resolved IEDs are determined for the most abundant ions, and the pressure induced changes in charge state as well as in kinetic ion energy are evaluated. The metal IEDs are fitted with shifted Maxwellian distributions, and the fitting parameters are analyzed. Introduction of gas reduced the amplitude of the ion signals and resulted in a decrease of the average charge state from 1.87 to 1.0. Similarly, the average energies decreased by approximately $70 \%$ in oxygen, from 258,177 , and $89 \mathrm{eV}$ for $\mathrm{Al}^{3+}, \mathrm{Al}^{2+}$ and $\mathrm{Al}^{1+}$, respectively, and even more in argon. The results indicate that the ion charge state and kinetic energy distributions can be influenced in a controlled manner by the choice of gases and their partial pressures.

\section{EXPERIMENTAL DETAILS}

Vacuum arc plasma was generated from a conical aluminum cathode with base and top diameters of 51 and $12 \mathrm{~mm}$, respectively, and a height of $38 \mathrm{~mm}$. The 
arc was initiated by a switched self trigger, ${ }^{16}$ and powered by a direct current (dc) arc supply, with resulting arc current of $35 \mathrm{~A}$. The cathode spots were confined to the cathode surface by a permanent ring magnet located behind the cathode. All measurements were performed after plasma filtering in a curved duct, see schematic of the experimental setup in Fig. 1. The distance from the cathode to the analyzer was $44 \mathrm{~cm}$. The magnetic field in the filter was generated by a coil of 640 turns and a current of $9 \mathrm{~A}$, with a resulting estimated field strength of 40 mT (a range generally employed for plasma filtering). Plasma characterization was carried out at base pressure $\left(1.8 \cdot 10^{-6}\right.$ Torr) using a Langmuir probe in the form of a $10 \mathrm{~mm}$ long $\mathrm{Pt}$ wire exposed to the plasma (at the position of the analyzer entrance). The plasma potential was found to be approximately $+3 \mathrm{~V}$ with respect to the anode (ground). The Langmuir probe was retracted for plasma analysis, where a mass-energy analyzer (PPM-422, Pfeiffer Vacuum) was used. This device can determine the plasma chemistry through mass-tocharge measurements at fixed energy, and IEDs through energy measurements at fixed mass-to-charge ratio. The entrance orifice of the analyzer was grounded, and all IEDs were measured with respect to ground. Data were collected at base pressure and after introduction of 1.8, 3.6, 7.4, 15.2, and $29.4 \mathrm{sccm}$ oxygen, corresponding to $0.5,1,2,4$, and 7 mTorr oxygen partial pressure, respectively, and after introduction of $2.7,5,10.1$, and $21.5 \mathrm{sccm}$ argon, corresponding to 1 , 1.9, 4, and 8 mTorr argon partial pressure, respectively (measured with an ion gauge). The raw data corresponding to the measured IEDs of the metal ions at base pressure are shown in Fig. 2. In order to represent smoothened IEDs 
without high frequency fluctuations in the distribution, Fourier components with frequencies higher than $\Delta t / n$ were removed, where $n$ is the number of data points considered at a time, and $\Delta t$ is the time interval between two adjacent data points. In the measured IEDs, every $1 \mathrm{eV}$ step was divided in 16 data points, and smoothening was done over a range of $5 \mathrm{eV}$. Note that the distributions in Fig. 2 are given as energy per charge, and hence, the energy scale is multiplied with 2 and 3 for $\mathrm{Al}^{2+}$ and $\mathrm{Al}^{3+}$, respectively, prior to calculation of the average energy, the average charge state, and the curve fitting procedure. Also note that in all figures presented below, no corrections were made with respect to ion acceleration in the sheath at the orifice; the corrections would correspond to approximately $Q \times 3 \mathrm{eV}$, where $Q$ is the ion charge state. The starting points of the IEDs measured at different pressures do not show any significant variation, and hence one can conclude that the plasma potential is approximately equal for all measurements.

\section{RESULTS AND DISCUSSION}

In a previous study, the effects of different geometric and magnetic field configurations on the charge-state-resolved IEDs were analyzed. ${ }^{6}$ It was found that the presence of a magnetic field drastically increased the ion energies. Here, the average ion energies at base pressure for $\mathrm{Al}^{1+}, \mathrm{Al}^{2+}$, and $\mathrm{Al}^{3+}$ were found to be approximately 90, 180, and $260 \mathrm{eV}$, respectively. This is consistent with previously reported measurements. ${ }^{6}$ The introduction of an oxygen partial 
pressure of up to $7 \mathrm{mTorr}$ resulted in decreased average energies of the metal ions and increased concentrations of the non-metal ions, the most abundant being $\mathrm{O}^{+}$, and $\mathrm{O}_{2}^{+}$. The charge-state-resolved average ion energies are shown in Fig. 3 , as a function of the resulting total pressure. The reproducibility of the measurements was demonstrated by repeating a series of measurements, which resulted in average energies of the metal ions with a deviation of $\pm 3 \%$ relative to what was previously measured.

Introduction of argon to a pressure of up to $8 \mathrm{mTorr}$ led to a stronger reduction of the ion energies as compared to the previously discussed interaction with oxygen, see Fig. 4. Furthermore, the intensity of the measured ion signals drastically reduced as the pressure was increased.

Although not shown in a figure, it should be mentioned that at an oxygen pressure of $1.0 \cdot 10^{-3}$ Torr, the peak intensity of the IEDs was comparable to the vacuum case. In contrast, when argon was used at the same pressure, the peak intensity was reduced to less than one tenth of the vacuum value. The decrease in intensity is evident in Fig. 4, where the increasing pressure resulted in a decrease of the IED intensity to insignificant levels, less than $1 / 1000$ of the vacuum intensity (and hence there are no data in the graph). This was first and foremost observed for $\mathrm{Al}^{3+}$, and at higher pressure also for $\mathrm{Al}^{2+}$. From these results one can conclude that, as expected, the pressure and the kind of gas affect on the average metal ion charge state, as shown in Fig. 5. For the same 
pressure, a difference in average charge state of up to 0.7 can be observed when comparing argon and oxygen

Within the hard sphere model, a slightly higher probability for collision in an argon environment, as compared to oxygen, is indicated under otherwise identical conditions. ${ }^{17}$ The energy transfer ${ }^{18}$ and the scattering angle ${ }^{19}$ in a collision depends on the mass of the constituents, and therefore argon (atomic weight $40 \mathrm{u}$ ) will have a somewhat larger impact upon a colliding metal ion than oxygen (molecular weight $32 \mathrm{u}$ ). However, the distinct differences observed between the results of the two gases indicate that a more detailed analysis is required, including differential cross sections for different elastic/inelastic collisions as well as their dependence on atomic/molecular species.

The interaction between plasma and gas may result in elastic and inelastic scattering. The latter includes impact excitation, ionization and charge transfer, possibly accompanied by molecule dissociation in an oxygen environment. The cross section for different charge transfer reactions strongly depends on the internal energy defect $\Delta E$, which is the difference in potential energies between pre-collision and after-collision particles. If $\Delta E$ is negative, the cross section is negligibly small. ${ }^{20}$ That is the case for $\mathrm{Al}^{1+}-\mathrm{Ar} / \mathrm{O}_{2}$ collisions, and $\mathrm{Al}^{2+}-\mathrm{O}_{2}$ collisions including molecule dissociation. ${ }^{17,21}$ It is therefore reasonable to assume that the decrease of the average charge state with increasing pressure, as shown in Fig. 5, is primarily due to charge exchange collisions between higher 
charged ions and the gas atom and molecules. Impact ionization collisions would increase the average charge state, and are therefore not discussed here.

The collision probability increases with pressure. ${ }^{22}$ Additionally, the cross section for most reactions show an energy dependence, as shown in the following examples: The cross section for resonant charge transfer for $\mathrm{Al}$ and $\mathrm{Ar}$ decreases by $30 \%$ as the energy increases from 0.1 to $10 \mathrm{eV},{ }^{23}$ and by $50 \%$ as the energy increases from 0.1 to $400 \mathrm{eV}^{18}$ Other data for $\operatorname{Ar}$ show a corresponding decrease of $\sim 45 \%$ as the energy increases from 7 to $178 \mathrm{eV}^{24}$ and furthermore, the cross section for elastic scattering of $\mathrm{Ar}$ in its parent gas decrease by more than $50 \%$ as the energy increase from 5 to $400 \mathrm{eV} .^{22}$ Under the assumption that the same trend is valid for all species in the present investigation, a decrease in energy will increase the charge transfer crosssection and hence increase the probability for charge transfer. The more pronounced decrease in intensity of ion signals and ion charge states in argon as compared to oxygen may be due to energy-dependent scattering cross sections and can not be understood based on the hard sphere model only. The oxygen case will be further discussed below.

Due to the drastic reduction in intensity for the argon case (and hence a much lower signal-to-noise ratio), further analysis was carried out for the oxygen experiment only. To gain additional information on the plasma parameters and their change with pressure, the smoothened IEDs of the metal ions were fitted by shifted Maxwellian distributions (SMD), as suggested by Kutzner et al. ${ }^{25}$ The 
approach was also adopted by Bilek et al. to describe the IEDs of $\mathrm{Ti}^{+}$in a nitrogen environment, ${ }^{14}$ and by Rosén et al. to investigate IEDs of $\mathrm{Al}$ ions in vacuum. ${ }^{6}$ The forward ion flux distribution of a SMD can be written as (units of energy and temperature in $\mathrm{eV}$ )

$$
f(E)=C_{s}\left[E-Q V_{p}\right] \cdot \exp \left[-\left(\sqrt{E-Q V_{p}}-\sqrt{E_{d i r}}\right)^{2} / T\right],
$$

where $C_{s}$ is a scaling constant, $V_{p}$ is the plasma potential with respect to the reference voltage of the analyzer (ground, here $0 \mathrm{~V}$ by convention), $E_{\text {dir }}$ is the directed energy (center-of-mass energy) of the ions, $Q$ the ion charge state, and $T$ is the ion temperature (or random energy). In a previous study, the broadening of averaged distributions due to the fluctuating character of momentary distributions was presented. ${ }^{26}$ It should therefore be stressed that the ion temperature is a formal assignment of a parameter to the time-averaged IED, and that the temperature (or width) of a momentary distribution is much smaller. Hence, $T$ will hereafter be referred to as simply the width of the distribution. The terms containing $Q$ are introduced to correct for the increase in ion energy due to the acceleration in the sheath at the analyzer entrance. In the curve fitting procedure, $\mathrm{C}_{\mathrm{s}}, E_{\text {dir, }}$ and $T$ were varied as to obtain the best agreement (corresponding to a minimized error function) with the measured and subsequently smoothened IEDs.

Smoothened IEDs with fitted SMDs are exemplified in Fig. 6. As can be seen, with increasing pressure, the distributions are shifted towards lower energies, and the concentration of lower charge states increases at the expense 
of higher ones. Fig. 6 also shows that the IEDs can be fitted well by SMDs. The fitting parameters for five different pressures are summarized in Fig. 7, together with the measured average energies of the metal ions (from Fig. 3) for comparison. The lines are drawn to guide the eye. Since only the forward flux of the ions is measured, the fitting parameters indicate the contribution of directed energy ( $\left.E_{\text {dir }}\right)$ which is equivalent to the center of mass energy, and width of the distribution $(T)$ to the average energy, $\left(E_{\text {average }}\right)$. For approximately the same width of the distribution, a higher directed energy corresponds to a higher average energy. Correspondingly, a broadening of a distribution at an unchanged directed energy would result in an increased average energy, since ions of high energy have a stronger weight in the average energy integral calculus than ions of low energy. Hence, the broad distributions (Fig. 7c) are the explanation for the observed difference between the average energy and the directed energy in Fig. $7 \mathrm{a}$ and $7 \mathrm{~b}$. Comparing the parameters of the different $\mathrm{Al}$ ions, one can see that the higher average energy of the higher charged ions is due to both a higher directed energy and a broader distribution. It has previously been suggested that the higher directed energy of the higher charged ions is primarily due to chargestate-dependent ion acceleration due to electric fields in the magnetized plasma. ${ }^{6}$ The difference in distribution widths may be explained by charge transfer, even at base pressure: Recent findings indicate that neutrals are present in cathodic arc plasmas, the main source being limited sticking and self-sputtering that occurs when energetic ions condense on substrates and chamber walls, especially at oblique incident angles. ${ }^{27}$ Collisions of ions with these metal neutrals will, in 
addition to a possible reduction in energy from elastic impacts, cause some of the higher charged ions to cascade down to lower charge states, most observable from a change in IED for $\mathrm{Al}^{3+}$. From an energy-dependent cross section for charge transfer ${ }^{23}$ one would expect an IED with a high energy tail, and a decreased maximum intensity of the distribution. Hence, the distribution will appear broader. Introduction of gas has the same effect on the IEDs, though more pronounced. As can be seen in Fig. 7c, the width of the distributions increase up to $1 \cdot 10^{-3}$ Torr. As previously discussed, an energy-dependent cross section for both elastic collisions $s^{22}$ and charge transfer reactions ${ }^{18,23}$ result in the high energy tail of the distributions being unchanged at moderate pressures. Hence, collisions (including charge transfer) mainly result in decreased maximum intensities (amplitudes) of the distributions, and increased populations of ions in the lower energy range, corresponding to distribution broadening. As the pressure is increased further, the mean free path decreases, ${ }^{22}$ resulting in frequent collisions of also the high energy ions, and hence a decrease in the distribution width (which is indeed observed in the graph at pressures above $1 \cdot 10^{-3}$ Torr). In Fig. $7 \mathrm{~d}$, the plasma potential times charge state is given. It can be seen that the plasma potential is independent of the gas pressure.

From the results presented above, one may expect narrower IED's, lower ion energies, and decreased ion signal intensities (most pronounced for higher charged ions) with further increased oxygen pressure. The latter is confirmed in Fig. 5, at the highest pressure presented here. Hence, it is possible to reduce the 
charge state distribution to include only singly charged ions, in argon as well as in oxygen, though at expense of ion signal intensity. This is of importance for the thin film growth process, enabling growth of alumina at well defined ion energies, which in turn may lead to better control of structure and composition of the resulting film.

\section{CONCLUSIONS}

Charge-state-resolved ion energy distributions (IEDs) were measured for aluminum cathodic arc plasmas operated in argon and oxygen atmospheres. The IEDs of the metal ions in oxygen could be well fitted by shifted Maxwellian distributions. Higher energies were observed for higher charged ions, with averages of 258,177 , and $89 \mathrm{eV}$ for $\mathrm{Al}^{3+}, \mathrm{Al}^{2+}$ and $\mathrm{Al}^{1+}$, respectively, which suggests acceleration by the electric fields that can exist in the magnetized plasma. Introduction of gas resulted in decreased energies, charge states, and ion signal intensities, which may be explained by scattering and charge transfer. These trends were most pronounced in argon, eventually resulting in metal ion

presence of $\mathrm{Al}^{1+}$ only, with an average energy of $19 \mathrm{eV}$. With increasing pressure, the average charge state decreased from 1.87 to 1.0 , showing that it is possible to obtain a plasma composition with a narrow charge-state distribution as well as a narrow IED. 


\section{ACKNOWLEDGMENTS}

The authors gratefully acknowledge the financial support granted by the Deutsche Forschungsgemeinschaft (DFG) within the project Schn 735/8-1, and by the U.S. Department of Energy under Contract No. DE-AC02-05CH11231 at Lawrence Berkeley National Laboratory. 


\section{REFERENCES:}

${ }^{1}$ A. Anders and G. Y. Yushkov, J. Appl. Phys. 91, 4824 (2002)

${ }^{2}$ E. Byon and A. Anders, J. Appl. Phys. 93, 1899 (2003)

${ }^{3}$ J. E. Greene, S. A. Barnett, J.-E. Sundgren, and A. Rocket, Ion Beam Assisted Thin Film Growth, edited by Itoh (Elsevier, Amsterdam, 1989), Chap. 5.

${ }^{4}$ A. A. Voevodin, J. G. Jones, and J. S. Zabinski, Appl. Phys. Lett. 78, 730 (2001)

${ }^{5}$ X. Wang, A. Kolitsch, A. Mueklich, F. Prokert, and W. Möller, J. Mat. Sci. Lett. $19,485(2000)$

${ }^{6}$ J. Rosén, A. Anders, S. Mráz, and J. M. Schneider, J. Appl. Phys. 97, 103306 (2005)

${ }^{7}$ R. N. Tarrant, M. M. M. Bilek, T.W.H. Oates, J. Pigott, and D.R. McKenzie, Surf. Coat. Technol. 156, 110 (2002)

${ }^{8}$ E. Oks, G. Yushkov, Proceedings of the International Symposium on Discharges and Electrical Insulation in Vacuum, Berkeley, 584 (1996)

${ }^{9}$ P. Spädtke, H. Emig, B. H. Wolf, and E. Oks, rev. Sci. Instrum. 65, 3113 (1994)

${ }^{10} \mathrm{~J}$. Rosén, A. Anders, L. Hultman, and J. M. Schneider, J. Appl. Phys. 94, 1414 (2003)

${ }^{11}$ I. Ivanov, H. Ljungcrantz, G. Håkansson, I. Petrov, and J.-E. Sundgren, Surf. Coat. Technol. 92, 150 (1997)

${ }^{12}$ L. Yang, J. Zou, and Z. Cheng, IEEE Trans. Plasma Sci. 25, 700 (1997)

${ }^{13}$ M. Chhowalla, Appl. Phys. Lett. 83, 1542 (2003)

${ }^{14}$ M. M. M. Bilek, P. J. Martin, and D. R. McKenzie, J. Appl. Phys. 83, 2965 (1998) 
${ }^{15}$ G. N. Strauss and H. K. Pulker, Proceedings of the $4^{\text {th }}$ International Conference on Coatings on Glass (Braunschweig, Germany, 2002)

${ }^{16}$ A. Anders, I. G. Brown, R. A. MacGill, and M. R. Dickinson, J. Phys. D: Appl. Phys. 31, 584 (1998)

${ }^{17}$ Handbook of Chemistry and Physics, $81{ }^{\text {st }}$ ed. (CRC, Boca Raton, 2000)

${ }^{18}$ B. Chapman, Glow Discharge Processes (Wiley, New York, 1980)

${ }^{19}$ E. W. McDaniel, Collision Phenomena in lonized Gases (Wiley, New York, 1964)

${ }^{20}$ J. Rosén, A. Anders, L. Hultman, and J. M. Schneider, J. Appl. Phys. 96, 4793 (2004)

${ }^{21}$ K. Honkala and K. Laasonen, Phys. Rev. Lett. 84, 705 (2000)

${ }^{22}$ M. A. Liberman and A. J. Lichtenberg, Principles of Plasma Discharges and Materials Processing (Wiley, New York, 1994)

${ }^{23}$ B. M. Smirnov, Phys. Scr. 61, 595 (2000)

${ }^{24}$ A. V. Phelps, J. Phys. Chem. Ref. Data, 20, 557 (1991)

${ }^{25}$ J. Kutzner and H. C. Miller, J. Phys. D: Appl. Phys. 25, 686 (1992)

${ }^{26}$ A. Anders, IEEE Trans. Plasma Sci. 27, 1060 (1999)

${ }^{27}$ A. Anders, Appl. Phys. Lett. 86, 6137 (2004) 


\section{FIGURE CAPTIONS:}

Figure 1: Schematic of the experimental setup.

Figure 2: Measured raw data corresponding to IEDs of the metal ions at base pressure.

Figure 3: Average ion energies vs total pressure (introduction of oxygen). The lines are drawn to guide the eye.

Figure 4: Average ion energies vs total pressure (introduction of argon). The lines are drawn to guide the eye.

Figure 5: Average metal ion charge state vs total pressure (introduction of argon/oxygen). The lines are drawn to guide the eye.

Figure 6: Smoothened charge-state-resolved IEDs of the metal ions at a) $1.9 \cdot 10^{-6}$ Torr b) $1 \cdot 10^{-3}$ Torr and c) $4 \cdot 10^{-3}$ Torr (introduction of oxygen), fitted with shifted Maxwellian distributions (SMDs).

Figure 7: Average energy and fitting parameters of SMDs vs total pressure (introduction of oxygen). The lines are drawn to guide the eye. 
Fig. 1, Rosén et al, JAP

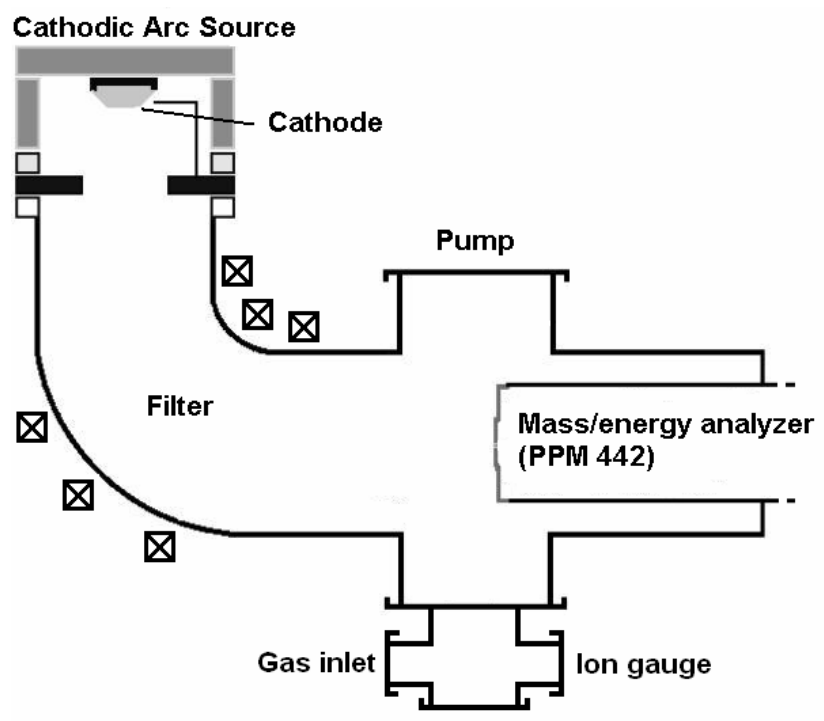


Fig. 2, Rosén et al, JAP

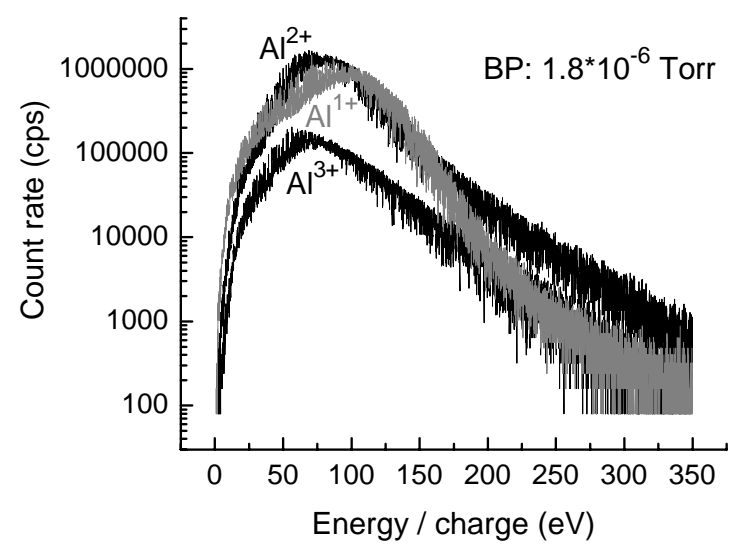


Fig. 3, Rosén et al, JAP

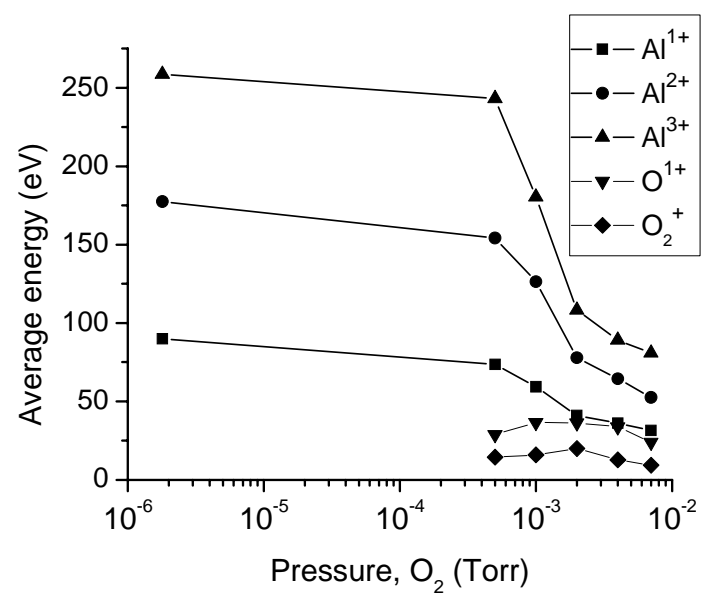


Fig. 4, Rosén et al, JAP

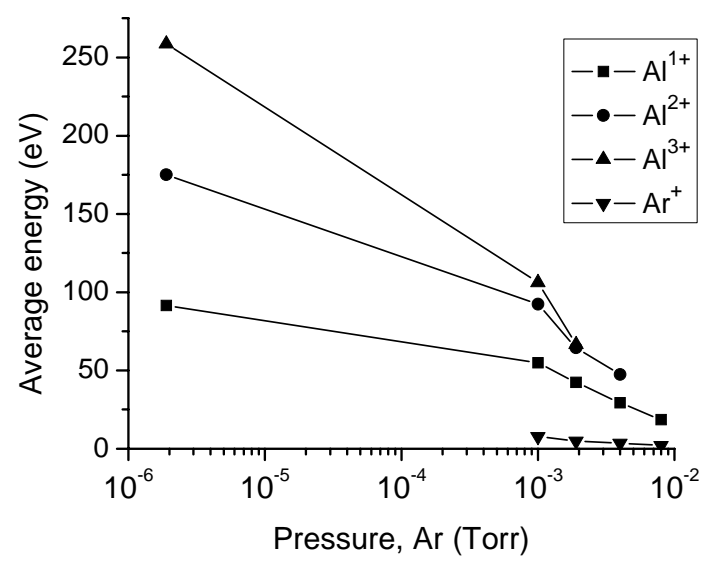


Fig. 5, Rosén et al, JAP

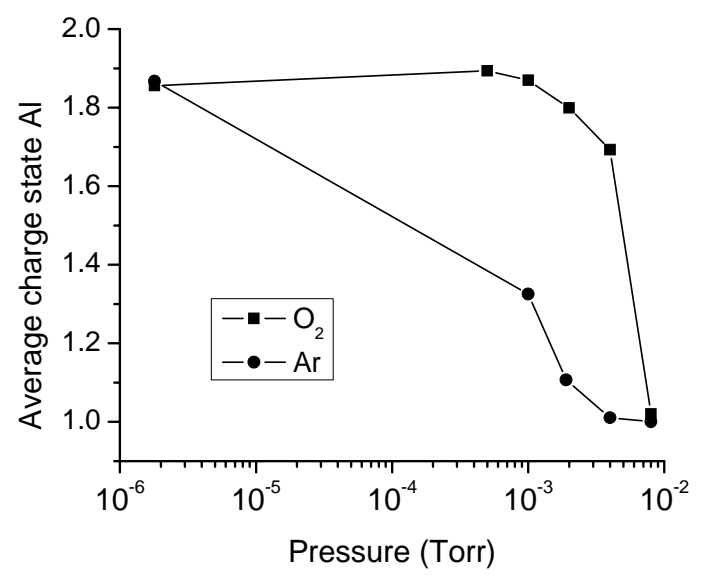


Fig. 6, Rosén et al, JAP

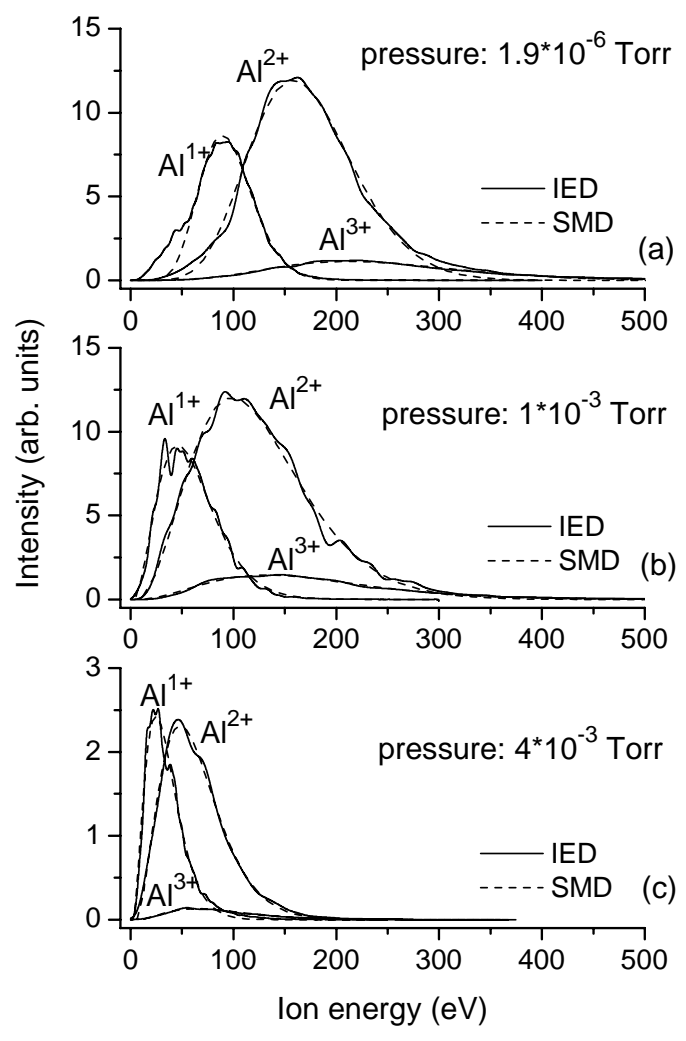


Fig. 7, Rosén et al, JAP
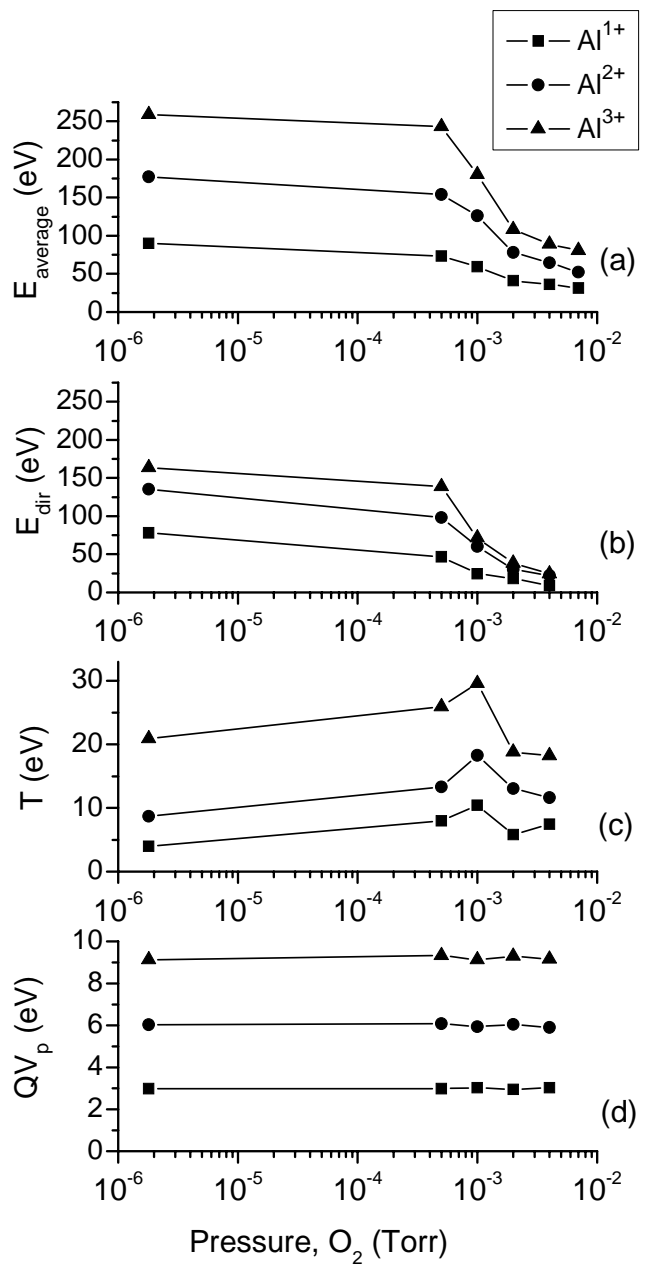